\title{
Universal Frequency Dependence of the Hopping AC Conductance in p-Ge/GeSi Structures in the Integer Quantum Hall Effect Regime
}

\author{
I. L. Drichko, ${ }^{1}$ A. A. Dmitriev, ${ }^{1,2}$ V. A. Malysh, ${ }^{1}$ I. Yu. Smirnov, ${ }^{1}$ \\ Y. M. Galperin,${ }^{3,1}$ H. von Känel, ${ }^{4}$ M. Kummer, ${ }^{4}$ D. Chrastina, ${ }^{5}$ and G. Isella ${ }^{5}$ \\ ${ }^{1}$ Ioffe Institute, 26 Politekhnicheskaya, 194021 St. Petersburg, Russia \\ ${ }^{2}$ Department of Nanophotonics and Metamaterials, ITMO University, \\ 49 Kronverksky Pr., 197101 St. Petersburg, Russia \\ ${ }^{3}$ Department of Physics, University of Oslo, 0316 Oslo, Norway \\ ${ }^{4}$ Laboratory for Solid State Physics, ETH Zurich, Otto-Stern-Weg 1, CH-8093 Zurich, Switzerland \\ ${ }^{5}$ L-NESS, Dipartimento di Fisica, Politecnico di Milano, \\ Polo Regionale di Como, Via Anzani 52, I-22100 Como, Italy
}

(Dated: December 13, 2018)

\begin{abstract}
The hopping ac conductance, which is realized at the transverse conductance minima in the regime of the integer Hall effect, has been measured using a combination of acoustic and microwave methods. Measurements have been made in the p-GeSi/Ge/GeSi structures with quantum wells in a wide frequency range $(30 \div 1200 \mathrm{MHz})$. The experimental frequency dependences of the real part of ac conductance $\sigma_{1}$ have been interpreted on the basis of the model presuming hops between localized electronic states belonging to isolated clusters. At high frequencies, dominating clusters are pairs of close states; upon a decrease in frequency, large clusters that merge into an infinite percolation cluster as the frequency tends to zero become important. In this case, the frequency dependences of the ac conductance can be represented by a universal curve. The scaling parameters and their magnetic-field dependence have been determined.
\end{abstract}

\section{INTRODUCTION}

Low-dimensional electronic systems have been objects of intense investigations for many years. The 2D electron gas in a quantizing transverse magnetic field is an informative object that exhibits different electric conduction mechanisms depending on the Landau level filling factors. The most known regimes include the integer and fractional quantum Hall effects 1 and the Wigner crystal. In the whole, a rich physical pattern is formed with details depending on the competition between the effects of structural disorder and the electronelectron interaction.

In this work, we are studying the hopping ac conductance of a 2D gas of holes, which is realized in p-Ge/GeSi structures at the minima of the integer quantum Hall effect (IQHE). In these regions, the electronic states are localized by disorder, and conduction occurs as hops between localized states. It is interesting that the physical patterns of the dc and ac conduction are substantially different in such a regime. In the former case, the hops occur over the states of the so-called percolation cluster that pierces the entire sample and terminates at the electrodes 2 . In the latter case, hopping occurs over the states of finite clusters isolated from one another (see, for example, review ${ }^{3}$ ). Therefore, comparative analysis of $\mathrm{dc}$ and ac conductions provides additional information.

The ac transport is studied using various experimental techniques among which contact-free methods occupy a special place. One of them is the acoustic method 4 based on the Rayleigh-type surface acoustic wave (SAW) propagating over the surface of a LiNbO3 piezoelectric substrate, to which the experimental sample is pressed (Fig. 1). In this case, a deformation wave in the substrate is accompanied with an electric field varying with the SAW frequency, which penetrates into the sample and interacts with charge carriers. As a result of such an interaction, the SAW intensity decreases (absorption) and the SAW velocity changes, which is registered by the detecting system. Then the complex ac conductance of the investigated sample is calculated from the experimentally measured SAW absorption coefficient and the change in the SAW velocity. It should be noted that in this method, the electric field polarization vector in the sample is parallel to the SAW wavevector, and the SAW itself is used only for introducing an electric field into the experimental sample by the contactless technique without deforming it.

A certain disadvantage of the acoustic method is the limited range of working frequencies, which is mainly determined by the configuration of interdigital transducers used for generating and detecting SAWs. Since analysis of the frequency dependence of the conductance makes it possible to study the mechanisms of localization of charge carriers in quantum size systems,,$\frac{5}{-7}$ it is important to perform measurements in a wide frequency range.

Such a possibility is ensured by the microwave contactless technique ${ }^{8}$ based on the use of a quasitransverse electromagnetic wave (quasi-TEM wave) propagating in a coplanar waveguide formed on the surface of insulating GaAs substrate (Fig. 2). The coplanar waveguide is prepared in the form of a meander for increasing the length over which charge carriers interact with the electric field of a quasi-TEM wave in the sample under investigation.

In this case, the experimental sample is pressed to the coplanar waveguide. Analogously to the electric field of the SAW in the acoustic method, the electric field of the quasi-TEM wave penetrates into the sample and interacts with charge carriers; as a result, the wave amplitude 

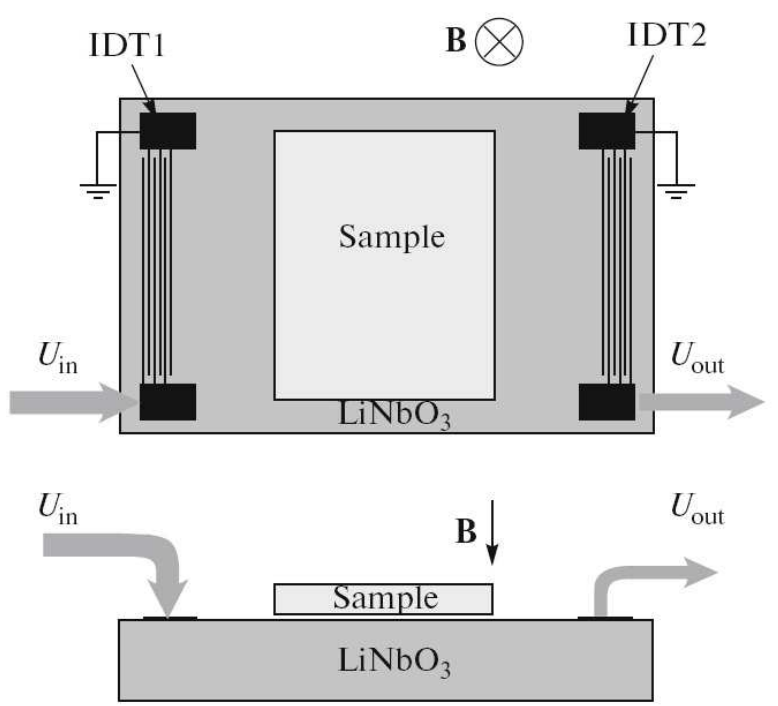

FIG. 1. Illustration the acoustic method. A SAW is generated by interdigital transducer 1 (IDT1) and is detected by IDT2 after the passage over the surface of the LiNbO3 piezoelectric substrate.

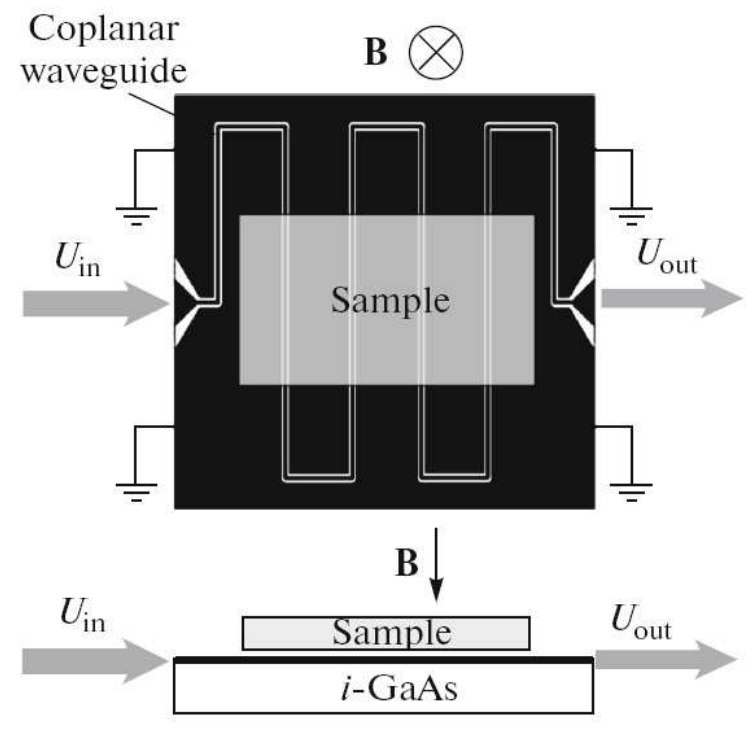

FIG. 2. Sketch of the microwave technique.

decreases and its phase changes. These quantities are subsequently used for determining the ac conductance of

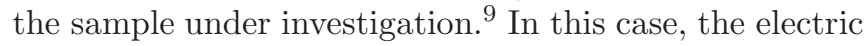
field polarization vector is directed in the same way as in the acoustic method, and the electric field of the quasiTEM wave is also introduced into the sample by the contactless method. The range of the working frequencies in the microwave method is wider and is in fact determined by the potentialities of the measuring instruments used in the experiment and not by the configuration of the coplanar waveguide itself. At the same time, the disadvantage of the microwave technique is that the absolute values of the ac conductance cannot be determined in view of specific features of this method.

This study aims at analysis of the low-temperature mechanisms of ac conduction in the Ge- and Si-based objects in a wide frequency range. For this purpose, the above-mentioned acoustic and microwave techniques were used. We employed the method of comparison of the results obtained using these two techniques, which was proposed in [10]; in this method, microwave measurements can be calibrated in the absolute values of conductance by comparing with the results of acoustic measurements in the accessible frequency range. It will be shown below that such a calibration must be performed in magnetic fields that correspond to the frequency-independent conductance maxima, which are determined by extended charge carriers.

\section{SAMPLES}

We have studied two p-Ge/SiGe samples - 1 with $13 \mathrm{~nm}$ quantum well with hole densities from $4 \times 10^{11} \mathrm{~cm}^{-2}$, and 2 - with $20 \mathrm{~nm}$ quantum well with and $p=6 \times 10^{11} \mathrm{~cm}^{-2}$, which were investigated in detail in [10]. These data will be used in the discussion of experimental results.

The structure of sample no. 1 is shown schematically in Fig. 3. It was obtained by chemical deposition from the vapor phase with the help of a low-energy plasma beam (LEPECVD) $\stackrel{11}{1}$ The active part of the sample is a 2D channel in a stressed Ge layer (of thickness $13 \mathrm{~nm}$ ), which is confined between SiGe layers with a Ge concentration of about $70 \%$. Modulated doping was performed by introducing diluted diborane $\left(\mathrm{B}_{2} \mathrm{H}_{6}\right)$ in the layers above and below the channel. The concentration and mobility of holes in the $2 \mathrm{D}$ channel at $T=1.7 \mathrm{~K}$ were $p=4 \times 10^{11} \mathrm{~cm}^{-2}$ and $\mu=4.4 \times 10^{4} \mathrm{~cm}^{2} /(\mathrm{V} \cdot \mathrm{s})$, respectively. The 2D Ge channel is stressed due to tensile strain under which the subband of heavy holes turns out to correspond to a higher energy. The energy gap between the subbands of light and heavy holes is so wide that only heavy holes participate in conduction in the entire accessible temperature range.

\section{EXPERIMENTAL RESULTS}

The SAW absorption coefficient and the velocity variations in sample no. 1 were measured in the temperature interval (1.74.2) $\mathrm{K}$ in magnetic fields up to $8 \mathrm{~T}$ in the SAW frequency range from 30 to $200 \mathrm{MHz}$.

Figures $4 \mathrm{a}$ and $4 \mathrm{~b}$ show the magnetic-field dependence of the SAW absorption coefficient, $\Delta \Gamma(B)=\Gamma(B)$ $\Gamma(0) \approx \Gamma(B)$ (since $\Gamma(0) \ll \Gamma(B))$ ) and the magneticfield dependence of the SAW velocity variation, $\Delta V(B) / V(0)=[V(B)-V(0)] / V(0) \quad(f=142 \quad \mathrm{MHz}$, $T=4.2 \mathrm{~K})$. It can be seen that oscillations of the absorption coefficient and SAW velocity appearing in the magnetic field correspond to Shubnikov de Haas 


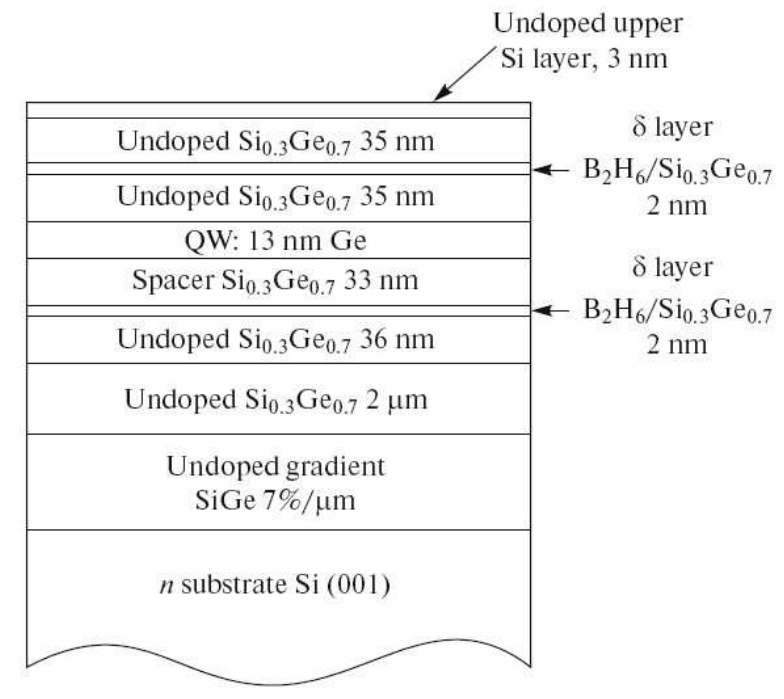

FIG. 3. Structure of sample no. 1.

oscillations below $3 \mathrm{~T}$ and to oscillations in the regime of the integer quantum Hall effect (IQHE) above $3 \mathrm{~T}$. Analogous curves were obtained for other frequencies and temperatures, as well as for sample no. 2 .

Dependences $\Gamma(B)$ and $\Delta V(B) / V(0)$ can be expressed in terms of the real $\left(\sigma_{1}\right)$ and imaginary $\left(\sigma_{2}\right)$ ac conductance components $\left(\sigma_{x x}^{A C}=\sigma_{1}-i \sigma_{2}\right)$ using the following expressions: $: \underline{12}$

$$
\begin{aligned}
& \Gamma=8.68 \frac{K^{2}}{2} q A \times \\
& \times \frac{4 \pi \sigma_{1} t(q) / \varepsilon_{s} V_{0}}{\left[1+4 \pi \sigma_{2} t(q) / \varepsilon_{s} V_{0}\right]^{2}+\left[4 \pi \sigma_{1} t(q) / \varepsilon_{s} V_{0}\right]^{2}}, \\
& A=8 b(q)\left(\varepsilon_{1}+\varepsilon_{0}\right) \varepsilon_{0}^{2} \varepsilon_{s} \exp [-2 q(a+d)] \text {, } \\
& \frac{\Delta V}{V_{0}}=\frac{K^{2}}{2} A \frac{1+4 \pi \sigma_{2} t(q) / \varepsilon_{s} V_{0}}{\left[1+4 \pi \sigma_{2} t(q) / \varepsilon_{s} V_{0}\right]^{2}+\left[4 \pi \sigma_{1} t(q) / \varepsilon_{s} V_{0}\right]^{2}}, \\
& b(q)=\left(b_{1}(q)\left[b_{2}(q)-b_{3}(q)\right]\right)^{-1}, \\
& t(q)=\left[b_{2}(q)-b_{3}(q)\right] / 2 b_{1}(q) \\
& b_{1}(q)=\left(\varepsilon_{1}+\varepsilon_{0}\right)\left(\varepsilon_{s}+\varepsilon_{0}\right)-\left(\varepsilon_{1}-\varepsilon_{0}\right)\left(\varepsilon_{s}-\varepsilon_{0}\right) e^{-2 q a}, \\
& b_{2}(q)=\left(\varepsilon_{1}+\varepsilon_{0}\right)\left(\varepsilon_{s}+\varepsilon_{0}\right)+\left(\varepsilon_{1}+\varepsilon_{0}\right)\left(\varepsilon_{s}-\varepsilon_{0}\right) e^{-2 q d} \text {, } \\
& b_{3}(q)=\left(\varepsilon_{1}-\varepsilon_{0}\right)\left(\varepsilon_{s}-\varepsilon_{0}\right) e^{-2 q a}+ \\
& +\left(\varepsilon_{1}-\varepsilon_{0}\right)\left(\varepsilon_{s}+\varepsilon_{0}\right) e^{-2 q(a+d)},
\end{aligned}
$$

where $K^{2}$ is the coefficients of the electromechanical coupling for lithium niobate; $q$ and $V_{0}$ are the wavevector and SAW velocity, respectively; $a$ is the gap between the piezoelectric lithium niobate substrate and the sample; $d$ is the depth of the location of the conducting channel (which is determined by technologists); $\varepsilon_{1}, \varepsilon_{0}$ and $\varepsilon_{s}$ are the dielectric constants for lithium niobate, vacuum, and
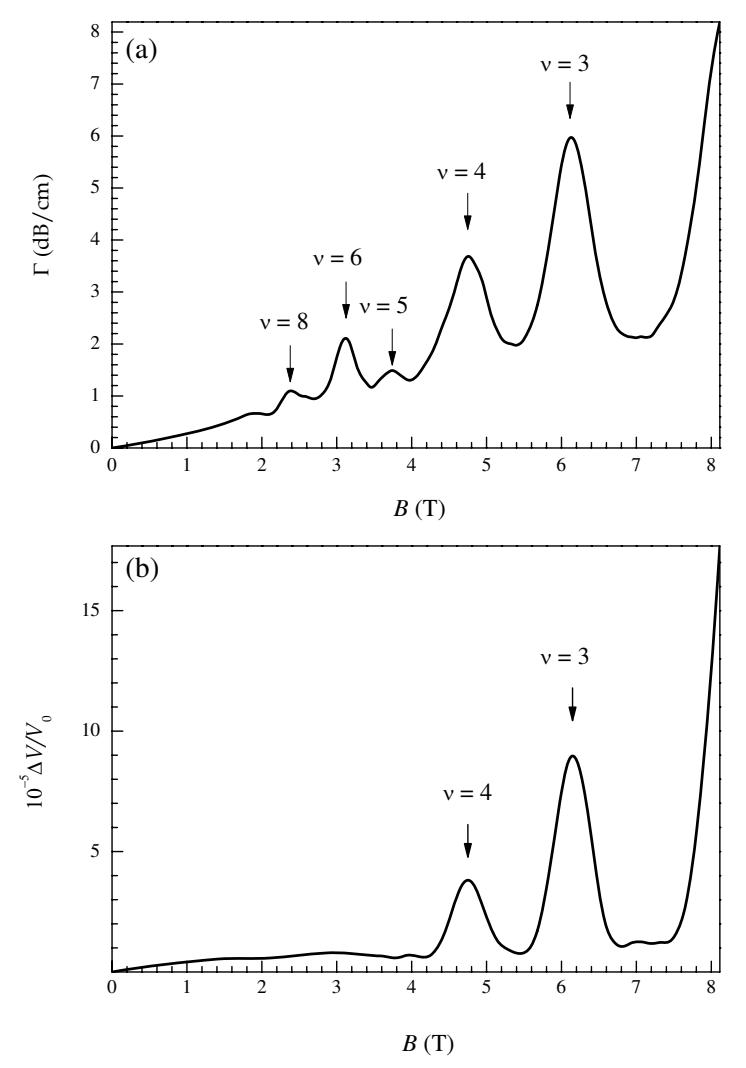

FIG. 4. Dependences $\Gamma(B)(\mathrm{a}), \Delta V / V(B)(\mathrm{b}), f=142 \mathrm{MHz}$, $T=4.2 \mathrm{~K}, \nu$ is the filling factor

the sample, respectively; and $b$ and $t$ are complex functions depending on $a, d, \varepsilon_{1}, \varepsilon_{0}$ and $\varepsilon_{s}$. The resultant dependence is shown in Fig. 5 .

The microwave measurements on sample no. 1 were made in magnetic fields up to $8 \mathrm{~T}$ in the frequency interval $100-1200 \mathrm{MHz}$ in the temperature range (1.74.2) K. Figure [6 shows the dependence of signal amplitude $U_{\text {out }}$ at the outlet of the coplanar waveguide on the magnetic field $(f=1130 \mathrm{MHz}, T=4.2 \mathrm{~K})$. Analogously to dependences $\Delta \Gamma(B)$ and $\Delta V(B) / V(0)$ measured using the acoustic technique, first the Shubnikovde Haas oscillations appear on the field dependence of $U_{\text {out }}$ (up to 3 $\mathrm{T}$ ), followed by oscillations in the IQHE regime (in fields above $3 \mathrm{~T}$ ).

The expression for calculating the real component $\sigma_{1}$ of the ac conduction in the microwave method has the form 8,9

$$
\sigma_{1}=-\frac{w}{Z_{0} l} \ln \left[\frac{U_{\text {out }}}{U_{\text {in }}}\right] \sqrt{1+\left[\frac{v_{\text {ph }}}{l \omega} \ln \left(\frac{U_{\text {out }}}{U_{\text {in }}}\right)\right]^{2}},
$$

where $U_{i n}$ is the signal amplitude at the input of the coplanar waveguide, $Z_{0}=50 \Omega$ is the characteristic impedance of the coplanar waveguide (without a sample), $l=5.3 \mathrm{~cm}$ is the length of the signal (serpentine) wire of the coplanar waveguide, $w=26 \mu \mathrm{m}$ is the width 


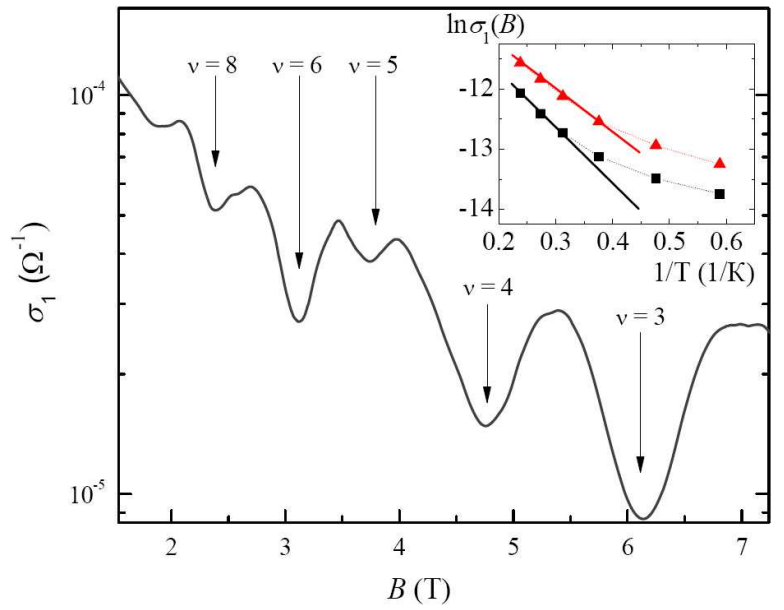

FIG. 5. Dependence $\sigma_{1}(B)$ for frequency $f=142 \mathrm{MHz}$ at $T=4.2 \mathrm{~K} ; \nu$ is the filling factor. The inset shows the dependence of $\ln \sigma_{1}(B)$ on inverse temperature at oscillation minima with filling factor $\nu=3$ (squares) and 4 (triangles).

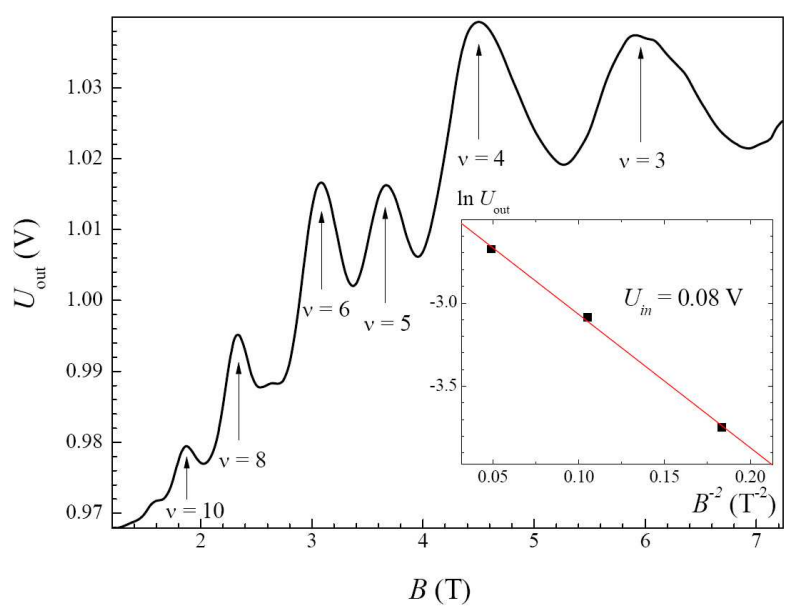

FIG. 6. Dependence $U_{\text {out }}(B)$ for frequency $f=1130 \mathrm{MHz}$ at $T=4.2 ; \nu$ is the filling factor; the inset shows the dependence of $\ln U_{\text {out }} \quad B^{-2}$.

of the gap between the signal wire and earthed conductor, $v_{p h}=c \sqrt{2 /\left(1+\varepsilon_{\mathrm{GaAs}}\right)}=1.14 \times 10^{8} \mathrm{~m} / \mathrm{s}$ is the phase velocity of the wave passing through the coplanar waveguide, and $\varepsilon_{\mathrm{GaAs}}=12.9$ is the dielectric constant of the i-GaAs substrate on which the coplanar waveguide has been formed.

It can be seen that for calculating $\sigma_{1}$, the value of $U_{i n}$ is required. It is extremely difficult to measure this quantity; for this reason, we have used the following assumptions for determining $U_{i n}$.

(1) The signal at the output of the coplanar waveguide is given by $U_{\text {out }}=U_{S}(B)+U_{L}$, where $U_{S}$ is the signal determined by the conductance of the sample and $U_{L}$ is the background signal characterizing the leakage through the coplanar waveguide. Our measurements have shown that the change in the phase of signal $U_{\text {out }}$ in a magnetic field is $20^{\circ}-50^{\circ}$. With such a change in the phase, the geometrical (vector) sum of signals $U_{S}$ and $U_{L}$ is very close to their algebraic sum. This enabled us to write signal $U_{\text {out }}$ in the form of the above-mentioned algebraic sum.

(2) In zero magnetic field, useful signal $U_{S}$ is equal to zero because the electric field of a quasi-TEM wave is screened by electrons almost completely for $B=0$. A noticeable $U_{S}$ signal appears only after the application of a magnetic field, when the conductance of the sample decreases.

(3) Background signal $U_{L}$ (at a preset frequency and temperature) does not change in a magnetic field and only produces a constant background of the output signal $U_{\text {out }}$. This is confirmed by experiments with coplanar waveguides.

Thus, taking into account assumptions (1)(3), we could reduce the calculation procedure to the following steps.

(i) Subtraction of the amplitude of background signal UL from the output signal $U_{\text {out }}$.

(ii) Determination of $U_{\text {in }}$ from the dependence of $\ln \left(U_{S}, \nu\right)$ on $B^{-2}$ (here $\nu$ is the filling factor for the Landau levels). This procedure was based on the following

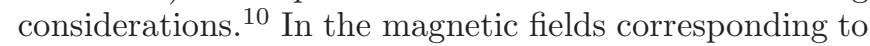
minima of IQHE oscillations, electrons are known to be localized, and ac conductance is of the hopping type. This fact is confirmed by the inset to Fig. ??, which shows the temperature dependence of the ac conductance for filling factors 3 and 4 . The flattening of the curves upon cooling indicates that the conduction mechanism becomes hopping indeed. In this case (see, for example, review [3] ), $\sigma_{1} \propto B^{-2}$ (to the logarithmic accuracy) in a strong magnetic field. If we disregard for simplicity the radicand in formula 2 (which is significant only at low frequencies, $\omega / 2 \pi \leq 300 \mathrm{MHz}$ ), this gives

$$
\sigma_{1}=-\frac{w}{Z_{0} l} \ln \left[\frac{U_{S}}{U_{i n}}\right]=-\frac{w}{Z_{0} l}\left[\ln \left(U_{S}\right)-\ln \left(U_{i n}\right)\right]
$$

whence

$$
\ln \left(U_{S}\right)=-\frac{Z_{0} l}{w} \sigma_{1}+\ln \left(U_{i n}\right) .
$$

Therefore, for $B \rightarrow \infty$, conductance $\sigma_{1} \rightarrow 0$ and $\ln \left(U_{S}\right) \rightarrow \ln \left(U_{i n}\right)$. The inset to Fig. ?? shows the dependence of $\ln \left(U_{S}, \nu\right) \quad B^{-2}$ for the maxima of the output signal or, in other words, for the conductance minima corresponding to occupation numbers $\nu=4,6,8$. The intersection of the line plotted using the linear approximation with the ordinate axis gives $\ln \left(U_{S}, \nu\right)=2.52$, which corresponds to $U_{\text {in }}=0.08 \mathrm{~V}$.

(iii) The determination of the real component of the high-frequency conductance by substituting the obtained value of $\left(U_{i n}\right)$ into formula (2). The result of such a calculation is shown in Fig. 7

At the peaks of oscillations, $\sigma_{1}(B)$ have an appreciable value in all magnetic fields $\left(\sigma_{1}>10^{-5} \Omega^{-1}\right)$; this corresponds to the conduction over extended states in the vicinity of the centers of the Landau levels, which is independent of frequency. It follows hence that the peaks 


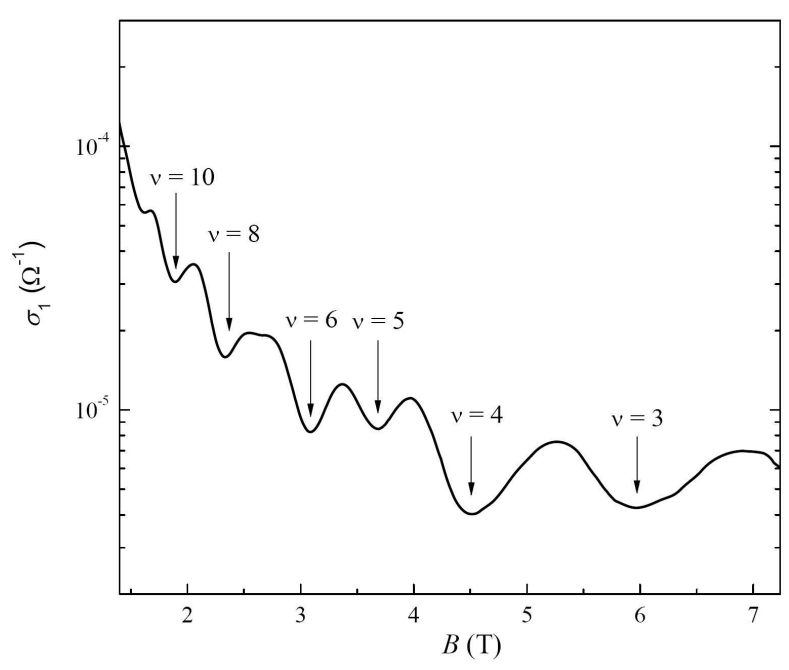

FIG. 7. Dependence $\sigma_{1}(B)$ for frequency $f=1130 \mathrm{MHz}$ at $T=4.2 \mathrm{~K} ; \nu$ is the filling factors.

of conductance oscillations, which are measured in the same magnetic field using different techniques, should coincide; actually, however, a discrepancy in these values is observed.

We think that this discrepancy is due to inaccuracy in determining conductance $\sigma_{1}$ with the help of the microwave method. The frequency independence of the conductance at the maxima enabled us to compare the results of the microwave and acoustic techniques. To this end, each $\sigma_{1}(B)$ dependence obtained by the microwave method was multiplied by correcting coefficient $K$, which was chosen so that the oscillation maxima determined using the acoustic and microwave techniques coincided. This precisely forms the additional (after stages (i)(iii)) procedure for processing of the results.

The results of this processing for different frequencies and $B=6.1 \mathrm{~T}$ at $T=4.2 \mathrm{~K}$ are shown in Fig. 8 . The ac conductance for $B<5.5 \mathrm{~T}$ is frequency-independent to within the experimental error.

The inset to Fig 8 confirms the correctness of the processing used: the curves measured with the help of these different methods at close frequencies in all magnetic fields coincide to within the experimental error after the superposition of the conductance peaks.

Figure 9] shows the frequency dependence of ac conductance $\sigma_{1}$ at the minimum corresponding to filling factor $\nu=3$ at $T=4.2 \mathrm{~K}$. It can be seen that for $f \leq 200 \mathrm{MHz}$, the frequency dependence is weak and $\sigma_{1}(\omega) \approx \sigma_{\mathrm{DC}}$. $f \geq 200 \mathrm{MHz}$, the behavior of the frequency dependence of 1 changes significantly. The interpretation of these results is given in the next section.

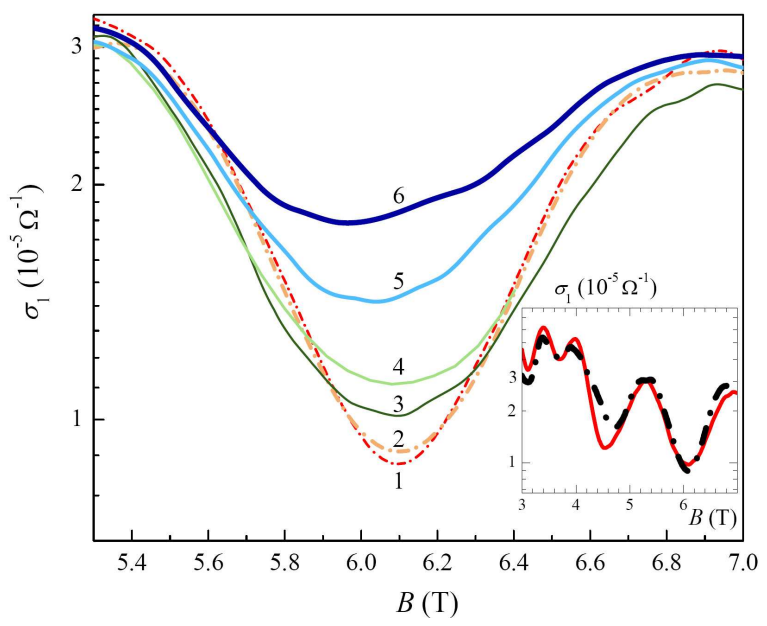

FIG. 8. Dependences $\sigma_{1}(B)$ for different frequencies: (1) $30 \mathrm{MHz}$ and (2) $142 \mathrm{MHz}$ (acoustic method); (3) $250 \mathrm{MHz}$, (4) $328 \mathrm{MHz},(5) 800 \mathrm{MHz}$, and (6) $1130 \mathrm{MHz}$ (microwave technique); $T=4.2 \mathrm{~K}$. The inset shows the $\sigma_{1}(B)$ dependences obtained by superposition of curves for $f \approx 220 \mathrm{MHz}$; solid and dashed curves correspond to the microwave and acoustic techniques.

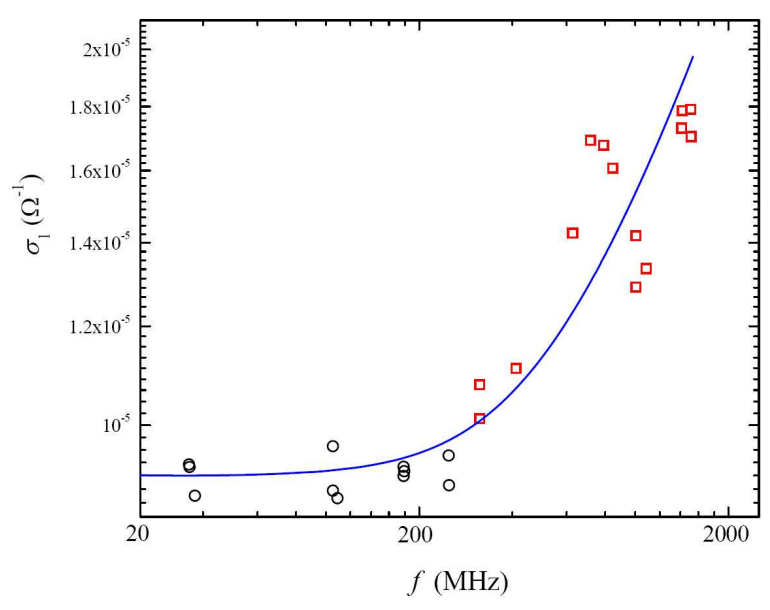

FIG. 9. Dependences $\sigma_{1}(f)$ at the minimum with filling factor $\nu=3$ at $T=4.2 \mathrm{~K} ; \bigcirc$ are the data obtained using the acoustic method; $\square$ correspond to the microwave technique. The solid scaling curve - is drawn in accordance with the theory described in Section 4.

\section{DISCUSSION}

\section{A. Absorption Mechanisms and Theoretical Models}

In the range of high frequencies, the ac hopping conduction is determined by the jump between the states belonging to the so-called close pairs in which the distances between the sites is much smaller than the mean distance between the sites. Therefore, absorption at high frequencies is determined by two site clusters. The dis- 
tance between the lower energy levels $E$ in such pairs is determined by the combination of the difference of onesite energies, $\varphi=\varphi_{1}-\varphi_{2}$, and the tunnel overlap integral $\Lambda(r)=\Lambda_{0} e^{-r / \alpha}$ (where $\alpha$ is the electron state localization length):

$$
E=\sqrt{\varphi^{2}+\Lambda^{2}} .
$$

There are two competing mechanisms of absorption, viz., the resonant and relaxation mechanisms (see, for example, [3]). In the former case, the wave attenuates due to direct absorption of ac quanta with $\hbar \omega=E$. This mechanism is important at high frequencies beyond our experimental frequency range.

The other (relaxation) mechanism is associated with modulation of occupancies of sites by the electric field of the wave, which varies with time. It noticeably depends on the population relaxation time, which can be represented in the form ${ }^{\underline{3}}$

$$
\frac{1}{\tau(E, r)}=\frac{1}{\tau_{\min }(E)}\left(\frac{\Lambda(r)}{E}\right)^{2} .
$$

Here, $\tau_{\min }(E)$ has the meaning of the minimal relaxation time for pairs with separation $E$ between energy levels. Power $Q$ absorbed by a pair can be written in the form

$$
Q=\frac{1}{2}\left|e \mathbf{E}_{0} \mathbf{r}\right|^{2} \frac{\omega^{2} \tau}{1+(\omega \tau)^{2}}\left(-\frac{\partial n_{o}}{\partial E}\right)
$$

The energy absorbed by a pair depends on two parameters $-E$ and $r\left(\right.$ via $\left.\Lambda(r) \propto e^{-r / \alpha}\right)$ ), and the total absorbed power is determined by the sum of the contributions from individual pairs. This summation can be expressed as the integral with respect to these parameters, which is evaluated taking into account their distribution functions. It turns out that pairs with $E \lesssim k T$ are important in this case. The result of summation over the pairs with different distances between the sites is determined by the exponential spread of tunnel integrals $\Lambda(r) \propto e^{-r / \alpha}$ in the case of a smooth distribution of distances between the centers. The final result depends on product $\omega \tau_{\min }(k T)$. In the most interesting case for our analysis, when

$$
\omega \tau_{\min }(k T) \ll 1
$$

the pairs with $\tau(k T) \approx \omega^{-1}$ (i.e., those in which the distributions of occupancies can reach equilibrium over a time on the order of the ac wave period) are most important. The characteristic size of such pairs is

$$
r_{\omega}=\alpha \ln \left[\Lambda_{o} / k T \sqrt{\omega \tau_{\min }(k T)}\right] \gg \alpha .
$$

For a 3D sample, the real part of the conductance turns out to be proportional to $\omega r_{\omega}^{3}$, which is usually expressed in the form $\sigma_{1} \propto \omega^{s}$, where exponent $s$ is several times smaller than unity and weakly depends on temperature $\underline{\underline{13}}$

It can be seen that upon a decrease in the frequency, the size of a characteristic pair increases, and pairs at low frequencies overlap to form large clusters (the so-called multiple hopping regime takes place). The size of a characteristic cluster in this case is determined by the stabilization of the population of sites during half-period $\pi / \omega$ of the wave. Therefore, the characteristic size of a cluster increases upon a decrease in frequency. With a further decrease in frequency, clusters merge into an infinitely large cluster responsible for static conduction, 2,14,15

There are several alternative models for describing the crossover from the ac conduction to dc conduction; the results of these models turn out to be close. In this study, we are using the Zvyagin model 15 formulated for the situation in which the spread in the rates of transitions between the centers is exponentially wide.

In this model, transitions between localized states $\lambda$ and $\lambda^{\prime}$ are characterized by the rates

$$
\Gamma_{\lambda \lambda^{\prime}}=\Gamma_{0} e^{-\eta_{\lambda \lambda^{\prime}}},
$$

where

$$
\eta_{\lambda \lambda^{\prime}}=\frac{2 r_{\lambda \lambda^{\prime}}}{\alpha}+\frac{\left|\varepsilon_{\lambda}-\varepsilon_{F}\right|+\left|\varepsilon_{\lambda^{\prime}}-\varepsilon_{F}\right|+\left|\varepsilon_{\lambda}-\varepsilon_{\lambda^{\prime}}\right|}{2 k T} .
$$

Like in the static problem on percolation conduction, two sites for the chosen value of $\Gamma$ are assumed to be coupled if

$$
\Gamma_{\lambda \lambda^{\prime}}>\Gamma .
$$

For an exponentially broad distribution of $\Gamma_{\lambda \lambda^{\prime}}$, the static conductance is determined by a certain critical value $\Gamma_{c}$, which corresponds to the formation of an infinite cluster.

Let us now choose a finite cluster $k$ in which the minimal transition rate satisfies the condition $\Gamma_{k}^{*}>\Gamma>\Gamma_{c}$. For a wide spread in transition rates, it is $\Gamma_{k}^{*}$ that determines the time of stabilization of equilibrium in the $k$ th cluster. Therefore, quasi-equilibrium is established in the cluster if

$$
\tau\left(\Gamma_{k}^{*}\right)<\omega^{-1} .
$$

For $\Gamma>\Gamma_{c}$, all clusters in the system are finite.

Let us now define quantity $\Gamma_{\omega}$ by the condition $\tau\left(\Gamma_{\omega}\right)=1 / \omega$; in this case, all clusters are quasiequilibrium, and relaxation losses occur only due to transitions between such clusters. The main idea is that for a wide spread in the transition rates, we can consider only the bottleneck, i.e., the transition at the lowest rate, which separates two quasi-equilibrium parts of a large cluster. This transition divides the cluster into two parts; as a result, a structure resembling a two-site system is formed; the sites of this system consist of parts of the initial cluster, in which transitions occur with relatively high rates. These parts play the role of the renormalized sites of the two-site model. Summation over such clusters is carried out on the basis of the known statistics of clusters in percolation systems 2,16 taking into account the fact that clusters with a relaxation time of occupancies on the order of $\omega^{-1}$ are important. 
Since the proposed procedure can be reduced to the renormalization of the properties of effective sites, it is not surprising that the theory predicts universal frequency dependences of ac conductance. According to [15], the result for the $3 \mathrm{D}$ case can be written in the form

$$
\frac{\sigma_{1}(\omega)}{\sigma_{\mathrm{DC}}} \ln ^{\xi}\left(\frac{\sigma_{1}(\omega)}{\sigma_{\mathrm{DC}}}\right)=\frac{\omega}{\omega_{S}} .
$$

where $\xi$ and

$$
\omega_{S}=\frac{\alpha}{C_{0} \eta_{c}^{\xi-1}} \sigma_{\mathrm{DC}}
$$

are the scaling parameters that generally depend on temperature. In the above expressions, $C_{0}$ is the capacitance per site and $\eta_{c}$ is the percolation threshold.

The derivation given in 15] does not contain specific features of 3D systems except that other critical indices appear in the 2D case. In addition, the dimension of the conductivity in the $2 \mathrm{D}$ case coincides with the dimension of the total conductivity. For our purpose, these differences are not very significant, because we analyze only the frequency dependences of the hopping ac conductance in the regime of the integer quantum Hall effect, treating critical index $\xi$ and frequency $\omega_{S}$ as fitting parameters. We do not consider here the complicated and interesting question concerning factor $\sigma_{\mathrm{DC}}$ in the IQHE regime, $\frac{17}{=}$ which affects frequency $\omega_{S}$.

The above scaling relations will be used below for analyzing the ac conductance at the oscillation minima, which corresponds to the regime of the integer quantum Hall effect, where the conduction is of the hopping type. Indeed, at low temperatures $(1.7 \mathrm{~K})$, the hopping nature of the ac conductance at the minima of oscillations is beyond any doubt, because the temperature dependence is flattened (see inset to Fig 5 ), and an increasing frequency dependence of $\sigma_{1}$ is observed even for $f>100 \mathrm{MHz}$. However, at higher temperatures $(4.2 \mathrm{~K})$ and low frequencies, the ac conductance is close to the static ac conductance, which depends on temperature in the activation manner (this is illustrated by straight lines in Fig. 5) because large clusters play the major role in this approximation. The frequency dependence corresponds to the typical behavior of hopping ac conductance only for $f>200 \mathrm{MHz}, \underline{\underline{5}}$

The curve plotted in Fig. 9] for sample no. 1 $(T=4.2 \mathrm{~K})$ corresponded to scaling relation (3) for parameters $\omega_{S}=4.0 \times 10^{9} \mathrm{~s}^{-1}$ and $\xi=0.5$. The value of $\sigma_{\mathrm{DC}}=9.1 \times 10^{-6} \Omega^{-1}$ was obtained by extrapolating the frequency dependence of $\sigma_{1}$ in the frequency range $f \leq 200 \mathrm{MHz}$.

It is convenient to trace the magnetic-field dependence of scaling parameters on sample no. 2, in which hopping conduction was observed at $T=1.7 \mathrm{~K}$ for several values of the magnetic field. Figure 10 shows the magneticfield dependence of the conductance of this sample for different frequencies.

The frequency dependence of the conductance of sample no. 2 in different magnetic fields is shown in Fig. 11.

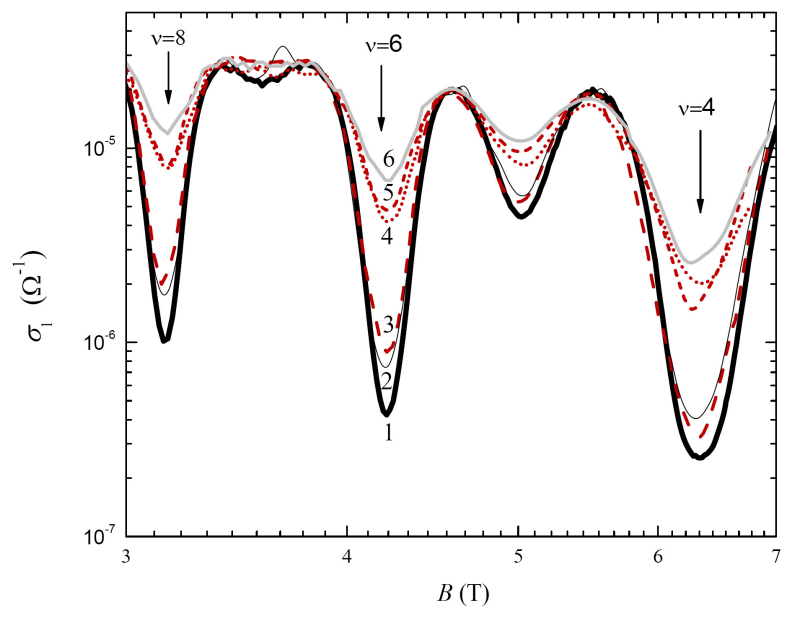

FIG. 10. Dependences of $\sigma_{1}$ on at different frequencies $f$, MHz: (1) 30, (3) 197 (acoustic method); (2) 148, (4) 585, (5) 906, and (6) 1191 (microwave technique); $T=1.7 \mathrm{~K}$; sample no. 2 .

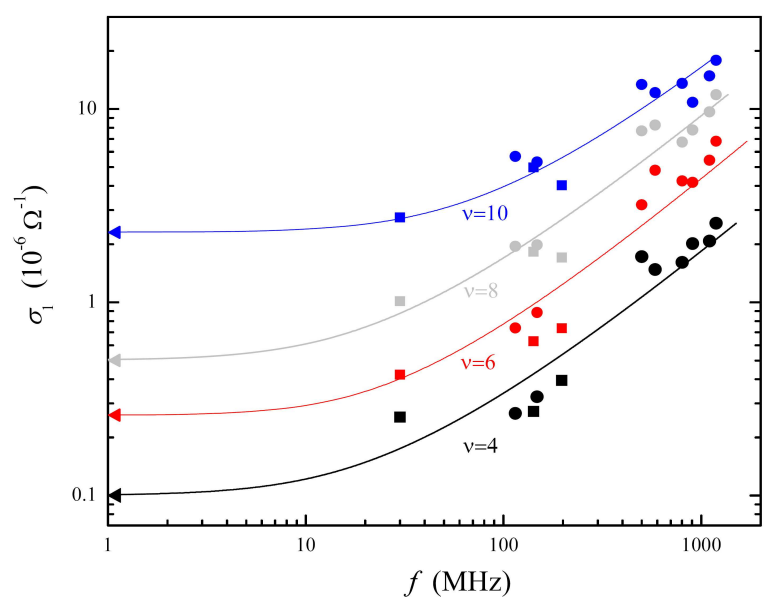

FIG. 11. Dependences $\sigma_{1}(f)$ at the minima of oscillations with $\nu=4,6,8,10$. Squares correspond to acoustic method, circles are the data obtained by microwave technique, and triangles correspond to $\sigma_{\mathrm{D}}, T=1.7 \mathrm{~K}$.

For plotting the curves in Fig. 11] we selected two parameters (scaling frequency $\omega_{S}=2 \pi f_{S}$ and dimensionless scaling parameter $\xi$ ). We used the value of $\sigma_{\mathrm{D}}$, which was determined for this sample in [18] at $T=1.7 \mathrm{~K}$.

The resultant values of the scaling parameters for sam-

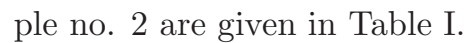

It can be seen from Table $\llbracket$ that the scaling parameters indeed depend on the magnetic field.

Scaling equation 3 can be used to express explicitly the dependence of ratio $\frac{\sigma_{1}}{\sigma_{\mathrm{DC}}}$ on $\frac{\omega}{\omega_{S}}$ :

$$
\frac{\sigma_{1}(\omega)}{\sigma_{\mathrm{DC}}}=\frac{\omega}{\omega_{S}}\left[\xi W\left(\frac{1}{\xi}\left[\frac{\omega}{\omega_{S}}\right]^{1 / \xi}\right)\right]^{-\xi},
$$

where $W(x)$ is the Lambert function. This dependence 
TABLE I. Scaling parameters.

\begin{tabular}{|c|c|c|c|c|}
\hline$\nu$ & $B(\mathrm{~T})$ & $\sigma_{\mathrm{DC}}\left(\Omega^{-1}\right)$ & $f_{S}(\mathrm{MHz})$ & $\xi$ \\
\hline 4 & 6.3 & $1 \mathrm{E}-7$ & 25 & 0.7 \\
6 & 4.22 & $2.6 \mathrm{E}-7$ & 32 & 0.6 \\
8 & 3.16 & $5 \mathrm{E}-7$ & 26 & 0.7 \\
10 & 2.53 & $2.3 \mathrm{E}-6$ & 88 & 0.7 \\
\hline
\end{tabular}

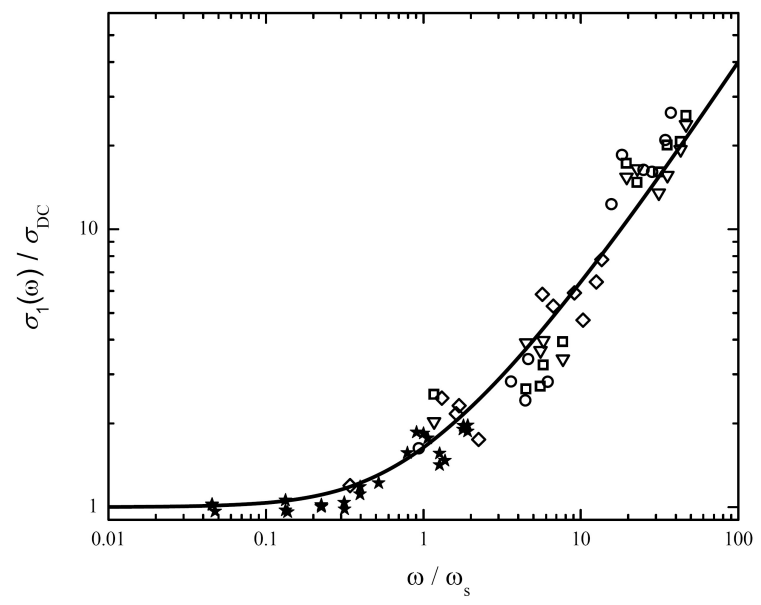

FIG. 12. Dependence of $\sigma_{1} / \sigma_{\mathrm{DC}}$ on $\omega / \omega_{S}: \star$ correspond to sample no. 1 ( $T=4.2 \mathrm{~K}), \square$ correspond to sample no. $2, \nu=4$; $\bigcirc$, sample no. $2, \nu=6 ; \nabla$, sample no. $2, \nu=8 ; \diamond$, sample no. $2, \nu=10$.

is plotted in Fig. 12 ,

It can be seen from the figure that all points for two different samples, different temperatures, and magnetic fields lie on the same curve, which proves the universality of the frequency dependence of the conductance in the hopping conduction conditions in this model.

It turned out that the value of $\xi$ weakly affects the frequency dependence: the change in $\xi$ from 0.4 to 0.7 leads to satisfactory agreement with experimental data at all oscillation minima (the curve in Fig. 12 was plotted for $\xi=0.7$ ). Since $\xi$ is a combination of critical indices, we can rightfully assume that this quantity weakly depends on the magnetic field. We have not detected such a dependence within the error of our experiment.

\section{CONCLUSIONS}

The high-frequency hopping conduction observed in magnetic fields corresponding to the conductance minima in the regime of the integer quantum Hall effect was measured in p-GeSi/Ge/GeSi structures with quantum wells in a wide frequency range $(30-1200 \mathrm{MHz})$. The measured frequency dependences are in agreement with the model presuming jumps between localized electron states belonging to isolated clusters. At high frequencies, predominant clusters are pairs of close states; upon a decrease in frequency, large clusters play the major role; as the frequency tends to zero, such clusters merge into an infinitely large percolation cluster. In this case, the frequency dependences of the ac conductance can be represented by a single universal curve (scaling). The scaling parameters and their magnetic-field dependence have been determined.

\section{ACKNOWLEDGMENTS}

The work of one of the authors (I.L.D.) was supported by the Presidium of the Russian Academy of Sciences.
1 The Quantum Hall effect, edited by R.E. Prange and S.M. Girvin (Springer-Verlag, New-York 1987).

2 B. I. Shklovskii and A. L. Efros, in: Electronic Properties of Doped Semiconductors (Springer-Verlag, Heidelberg) 1984.

3 Y. M. Galperin, V. L. Gurevich, D. A. Parshin, in: B. Shklovskii, M. Pollak (Eds.), Hopping Transport in Solids, Elsevier, NY, 1991.

4 A. Wixforth, J. Scriba, M. Wassermeier, J. P. Kotthaus, G. Weimann, and W. Schlapp, Phys. Rev. B 40, 7874 (1989).

5 I. L. Drichko, A. M. Diakonov, I. Yu. Smirnov, Y. M. Galperin, and A. I. Toropov, Phys. Rev. B 62, 7470 (2000).

6 I. L. Drichko, A. M. Diakonov, I. Y. Smirnov, G. O. Andrianov, O. A. Mironov, M. Myronov, D. R. Leadley, and T. E. Whall, Phys. Rev. B 71, 045333 (2005).

7 I. L. Drichko, A. M. Diakonov, V. I. Kozub, I. Yu. Smirnov, Yu. M. Galperin, A. I. Yakimov, A. I. Nikiforov, Physica E: Low-dimensional Systems and Nanostructures 26, 450 (2005).
${ }^{8}$ L. W. Engel, D. Shahar, Ç. Kurdak, and D. C. Tsui, Phys. Rev. Lett. 71, 2638 (1993).

9 A. Endo, T. Kajioka, and Y. Iye, J. Phys. Soc. Jpn. 82, 054710 (2013).

10 I. L. Drichko, A. M. Diakonov, V. A. Malysh, I. Yu. Smirnov, Y. M. Galperin, N. D. Ilyinskaya, A. A. Usikova, M. Kummer, and H. von Känel, J. Appl. Phys. 116, 154309 (2014).

11 C. Rosenblad, H. R. Deller, A. Dommann, T. Meyer, P. Schroeter, and H. von Känel, J. Vac. Sci. Technol. A 16, 2785 (1998).

12 V. D. Kagan, Fiz. Tech. Polupr. 31, 478 (1997) [V. D. Kagan, Semiconductors 31, 470 (1997)].

13 B. I. Shklovskii and A. L. Efros, ZhETF 81, 406 (1981) [Sov. Phys. JETP 54, 218 (1981)].

14 J. C. Dyre and T. B. Schrøder, Rev. Mod. Phys. 72, 873 (2000).

15 I. Zvyagin, AC Hopping Transport in Disordered Materials, in: Charge transport in disordered solids with applications in electronics, John Wiley \& Sons, 2006. 
16 D. Stauffer and A. Aharony, Introduction to Percolation Theory, Taylor and Francis, London, 1992.

17 D.G. Polyakov and B.I. Shklovskii, Phys. Rev. Lett. 70, 3796 (1993); Phys. Rev. B 48, 1167 (1993); Phys. Rev.
Lett. 74, 150 (1995) ibid 73, 1150 (1994).

18 H. von Känel, M. Kummer, G. Isella, E. Müller, T.Hackbarth, Appl. Phys. Lett. 80, 2922 (2002). 